\title{
Challenges to Digital Dentistry in Dental Schools
}

Ilser Turkyilmaz ${ }^{1}$, Samantha Lakhia ${ }^{2}$

Keywords: Curriculum, Digital dentistry, Infrastructure, Intraoral scanner, Student.

The Journal of Contemporary Dental Practice (2019): 10.5005/jp-journals-10024-2734

With the new era of rapidly expanding technology, digital dentistry promises many opportunities such as grading standardization, access to student self-assessment, less material waste, a more hygienic clinical protocol, and the potential for greater accuracy and predictability in clinical outcomes. ${ }^{1-3}$ Despite these tremendous advantages, some challenges such as cost, training, and infrastructure still remain, which prevent digital dentistry from becoming the new standard in dental institutions. ${ }^{2,4}$

In the dental school setting, digital dentistry promises greater opportunities for students to assess their skill sets without direct faculty supervision, more objective evaluation and grading of preclinical assessments, and the potential for improved clinical outcomes. The major barriers that remain are cost of digital dentistry hardware such as intraoral scanners and software, which may have to be updated in few years due to advancements in technology. ${ }^{5}$ Additionally, recruiting and retaining well-trained faculty to take on roles in digital dentistry utilization and instruction is crucial. In their absence, digital dentistry advances become useless, as the cornerstone of the technology is its proper utilization. Students who receive inadequate instruction on digital dentistry technology gain no benefit in terms of preclinical self-assessment and enhanced assessment of clinical outcomes. Faculty, who are more experienced in traditional dental methods and practising dentistry by using only those techniques, may not be willing or able to quickly adapt to the new technology, especially in a capacity where they will adequately be able to pass on these skills to students.

Managing curriculum to accommodate instruction on digital dental technologies also becomes essential. ${ }^{4}$ Due to the crowded curriculum and limitations in the ability to expand course load, the preclinical curriculum emphasizing techniques on impression taking and stone cast fabrication will likely face displacement. Alteration in the clinical protocol in favor of digital techniques will decrease material waste and establish a more hygienic method of obtaining impressions. Completely displacing instruction on traditional dental procedures in favor of procedures associated with digital dentistry may pose a substantial risk. Because, ultimately, dental students who were trained with exclusively digital dentistrydependent techniques may have a disadvantage as they enter a professional workplace, which may not have adopted the new technology to which they are accustom. Today, the integration of digital dentistry concepts with traditional dental procedures may be more practical. $^{2}$ \begin{tabular}{l} 
1'Department of Prosthodontics, New York University College of \\
Dentistry, New York, USA \\
${ }^{2}$ New York University College of Dentistry, New York, USA \\
Corresponding Author: Ilser Turkyilmaz, Department of Prosthodon- \\
tics, New York University College of Dentistry, New York, USA, Phone: \\
+1-212-992-7181, e-mail: ilserturkyilmaz@yahoo.com \\
How to cite this article: Turkyilmaz I, Lakhia S. Challenges to Digital \\
Dentistry in Dental Schools. J Contemp Dent Pract 2019;20(12):1361. \\
Source of support: Nil \\
Conflict of interest: None \\
\hline \hline
\end{tabular}

With patient privacy and protection remaining at the forefront, safely storing and sharing digital patient records and photos while still complying with the Health Insurance Portability and Accountability ACT (HIPAA) and the Family Educational Rights and Privacy Act (FERPA) is critical in the United States. ${ }^{2}$ With the rapid technological expansion in dentistry, infrastructure remodeling is even more critical than before. The use of data storage servers and external operating systems capable of handling the high volume of patient information safely for utilization by multiple clinicians and external laboratories becomes necessary.

It is important for institutions to recognize the challenges in advance of digital dentistry incorporation and build a solid foundation and infrastructure prior to introducing these technologies as a mainstream institutional standard.

\section{References}

1. Greany TJ, Yassin A, Lewis KC. Developing an all-digital workflow for dental skills assessment: part II, surface analysis, benchmarking, and grading. J Dent Educ 2019;83(12):1314-1322. DOI: 10.21815/JDE.019.133.

2. Cooper LF. Digital technology: impact and opportunities in dental education. J Dent Educ 2019;83(4):379-380. DOI: 10.21815/JDE.019.042.

3. Reiss B. Editorial: changing times: a new dynamic in digital dentistry. Int J Comput Dent 2019;22(3):211-213.

4. Afshari FS, Sukotjo C, Alfaro MF, et al. Integration of digital dentistry into a predoctoral implant program: program description, rationale, and utilization trends. J Dent Educ 2017;81(8):986-994. DOI: 10.21815/ JDE.017.050.

5. Resnick CM, Doyle M, Calabrese CE, et al. Is it cost effective to add an intraoral scanner to an oral and maxillofacial surgery practice? J Oral Maxillofac Surg 2019;77(8):1687-1694. DOI: 10.1016/j.joms.2019.03.011.

(O) The Author(s). 2019Open Access This article is distributed under the terms of the Creative Commons Attribution 4.0 International License (https://creativecommons. org/licenses/by-nc/4.0/), which permits unrestricted use, distribution, and non-commercial reproduction in any medium, provided you give appropriate credit to the original author(s) and the source, provide a link to the Creative Commons license, and indicate if changes were made. The Creative Commons Public Domain Dedication waiver (http://creativecommons.org/publicdomain/zero/1.0/) applies to the data made available in this article, unless otherwise stated. 